\title{
Hypothesis: Transposons (TEs) may present a mechanism of adaptation to selective pressure of trauma and its inheritance
}

Catarina Cunha ${ }^{1^{*}}$

${ }^{1}$ Emotional Brain Institute, Nathan Kline Institute, Orangeburg, NY, USA

${ }^{*}$ Correspondence: Catarina Cunha, Emotional Brain Institute, Nathan Kline Institute, Orangeburg, NY, USA E-mail: catarina.cunha@nki.rfmh.org

There are numerous studies exploring mechanisms of adaptation to selective pressure in various organisms, in which many hypothesize it being a predominant force in evolution ${ }^{1-}$ 3. Although adaptation is a basic and crucial mechanism affecting biology as a whole, we still remain in the dark about it. One aspect regarding selective pressure and how it affects us over generations is stress and trauma, which afflict most of us at some point of our lives (89\% of Americans) ${ }^{4}$. Numerous studies suggest that extreme environmental factors, such as trauma, change how DNA is being used in organisms to produce functioning proteins, which is supposed to play an important role, among other things, in neural plasticity. This form of direct adaptation to selective pressure is not realized through random classic mutations, and therefore presents a fast tool set for an organism to integrate environmental information, which is supposed to be passed down to next generations. While epigenetic mechanisms, such as DNA methylation, are involved in adaptation in one organism ${ }^{5}$, this is not a likely system to pass down information of adaptation to next generations for two reasons:

1) There are no known mechanisms how trauma that cause changes in neurons would affect fully formed germline cells before fertilization through methylation.

2) Even if germline cells would be modulated by trauma through methylation, these marks would most likely not survive to a significant enough extend, because of the various stages of methylation resetting mechanisms after fertilization and throughout development. ${ }^{6}$

An alternative system by which trauma adaptation could be transmitted to next generations, would be that of transposable elements (TEs). Traditionally they have been 
considered as junk DNA, which means that although representing a high proportion of genomes (ca. $45 \%$ ) no cellular functions could be detected. However, recently it has become evident that TE-derived DNA sequences not only play a fundamental role during evolution in all organisms, but that TEs are also implicated in the origin and evolution of various disorders ${ }^{7}$. Therefore, one could hypothesize, that TEs play a crucial role in adaptation in general, and especially to trauma ${ }^{8-10}$ considering the following facts:

1. TEs play an important role in the structural evolution of genomes through generation of various types of re-arrangements ${ }^{9,11}$.

2. TEs donate regulatory sequences that control expression of nearby genes ${ }^{11-14}$.

3. TEs become incorporated into coding sequences at the transcript level ${ }^{15-18}$.

4. TEs have their genes recruited by host genomes for key functions ${ }^{19}$.

5. TEs control expression levels, dependent upon circumstances: In the study, "High Rate of Recent Transposable Element-Induced Adaptation in Drosophila melanogaster", adaptations caused by transposable elements were detected. It was shown, that most of the TEs were located in introns, and that they caused significant differences in gene expression between the population in Africa and other parts of the world. The climate induced genetic adaptation, was traced back to four TEs that enabled the organisms to acclimate their gene expression to a new selective pressure ${ }^{20}$.

6. We hypothesize that TEs are the right candidates for trans-generational information transfer, because of their characteristics being selfish DNA or molecular parasites. TEs "want" to be perpetuated in genomes over generations. Supporting this theory, the activity of TEs were shown to be prominent in the germline and during embryonic development ${ }^{21-23}$.

7. The next results show that TEs have what is needed to survive embryo development: Engineered L1 retro-transposition assays have demonstrated that L1 can move in different cell types, suggesting that the minimal host factors required for retro-transposition are present in cells. Work in iPSCs has revealed that, upon reprogramming, $L 1$ expression is reactivated with respect to the parental cells, by methylation changes in the L1 promoter. In sum, different studies suggest 
that heritable L1 insertions can accumulate during human early embryonic development ${ }^{21,24}$.

On a general note, it was shown that using viral mechanisms to store and transfer information in neurons through Arc, is a common strategy in organisms. Arc has been demonstrated to be crucial for long term memory. Furthermore, Arc was labeled as the "master regulator" of synthesis-dependent forms of synaptic plasticity ${ }^{25-27}$.

Taken together these traits would be important for effective information transfer, to next generations. TE mechanisms make a strong case for being predominantly implicated in transmitting trans-generational adaption mechanisms derived from trauma.

\section{Proposal of experiments to test our hypothesis on human saliva samples over generations:}

Transposons (TE) are known to be up-regulated and more active during stress. They also occur highly in telomeres ${ }^{28}$, which is known to be affected by stress ${ }^{29}$. TE down-regulation is an anti-stress regulatory mechanism and is regulated mostly by methylation systems (DNMT3s), but also microRNAs play an important role 28 .

Our suggestion is to screen for TEs by using next-generation DNA sequencing searching for polymorphic mobile element insertions (pMEls) or even detecting de novo MEls in saliva from humans in at least 2 generations of families that went through trauma and families with comparatively low trauma. Different methods have been developed for the identification of MEls using high-throughput sequencing. MEls can be analyzed directly from whole-genome sequencing data. Regarding data analysis, in deep sequencing, the goal is to detect polymorphic or de novo MEls by a computational analysis. Different algorithms have been developed to find MEls, and two are the principal strategies to do it: "paired-end-reads mapping" and "split-read mapping".

These studies would provide unprecedented details about inheritance and gene expression patterns, and how they relate to the development and/or establishment of 
adaptation. Additionally, with the technology of next generation sequencing and rapid genome and transcriptome analyses, the dynamic nature of TEs and their role in intraand inter-individual variability may be elucidated. These methods enable us to dissect TE activity at a high resolution, which will lead to a better understanding of TEs mechanisms and their impact on human health. In summary, we are only beginning to scratch the surface of the roles that TE mechanisms play in our system, development, function, inheritance, and the roles that TEs play in pathologic states.

1 Wright, S. I. et al. The effects of artificial selection on the maize genome. Science $\mathbf{3 0 8}$, 1310-1314, doi:10.1126/science.1107891 (2005).

2 Andolfatto, P. Adaptive evolution of non-coding DNA in Drosophila. Nature 437, 11491152, doi:10.1038/nature04107 (2005).

3 Shapiro, J. A. et al. Adaptive genic evolution in the Drosophila genomes. Proc Natl Acad Sci U S A 104, 2271-2276, doi:10.1073/pnas.0610385104 (2007).

4 Kilpatrick, D. G. et al. National estimates of exposure to traumatic events and PTSD prevalence using DSM-IV and DSM-5 criteria. Journal of traumatic stress 26, 537-547, doi:10.1002/jts.21848 (2013).

5 Schulz, L. C. The Dutch Hunger Winter and the developmental origins of health and disease. Proceedings of the National Academy of Sciences 107, 16757, doi:10.1073/pnas.1012911107 (2010).

6 Seisenberger, S. et al. Reprogramming DNA methylation in the mammalian life cycle: building and breaking epigenetic barriers. Philosophical transactions of the Royal Society of London. Series B, Biological sciences 368, 20110330-20110330, doi:10.1098/rstb.2011.0330 (2013).

7 Reilly, M. T., Faulkner, G. J., Dubnau, J., Ponomarev, I. \& Gage, F. H. The role of transposable elements in health and diseases of the central nervous system. The Journal of neuroscience : the official journal of the Society for Neuroscience 33, 17577-17586, doi:10.1523/JNEUROSCI.3369-13.2013 (2013).

8 Kidwell, M. G. \& Lisch, D. R. Perspective: transposable elements, parasitic DNA, and genome evolution. Evolution 55, 1-24 (2001).

9 Kazazian, H. H., Jr. Mobile elements: drivers of genome evolution. Science 303, 16261632, doi:10.1126/science.1089670 (2004).

10 Biémont, C. \& Vieira, C. Junk DNA as an evolutionary force. Nature 443, 521, doi:10.1038/443521a (2006).

11 Hughes, J. F. \& Coffin, J. M. Evidence for genomic rearrangements mediated by human endogenous retroviruses during primate evolution. Nat Genet 29, 487-489, doi:10.1038/ng775 (2001).

12 Jordan, I. K., Rogozin, I. B., Glazko, G. V. \& Koonin, E. V. Origin of a substantial fraction of human regulatory sequences from transposable elements. Trends Genet 19, 68-72 (2003). 
13 Marino-Ramirez, L., Lewis, K. C., Landsman, D. \& Jordan, I. K. Transposable elements donate lineage-specific regulatory sequences to host genomes. Cytogenet Genome Res 110, 333-341, doi:10.1159/000084965 (2005).

14 Lowe, C. B., Bejerano, G. \& Haussler, D. Thousands of human mobile element fragments undergo strong purifying selection near developmental genes. Proc Natl Acad Sci U S A 104, 8005-8010, doi:10.1073/pnas.0611223104 (2007).

15 Lipatov, M., Lenkov, K., Petrov, D. A. \& Bergman, C. M. Paucity of chimeric genetransposable element transcripts in the Drosophila melanogaster genome. BMC Biol 3, 24, doi:10.1186/1741-7007-3-24 (2005).

16 Nekrutenko, A. \& Li, W. H. Transposable elements are found in a large number of human protein-coding genes. Trends Genet 17, 619-621 (2001).

17 Gotea, V. \& Makalowski, W. Do transposable elements really contribute to proteomes? Trends Genet 22, 260-267, doi:10.1016/j.tig.2006.03.006 (2006).

18 Piriyapongsa, J., Rutledge, M. T., Patel, S., Borodovsky, M. \& Jordan, I. K. Evaluating the protein coding potential of exonized transposable element sequences. Biology direct 2, 3131, doi:10.1186/1745-6150-2-31 (2007).

19 Volff, J. N. Turning junk into gold: domestication of transposable elements and the creation of new genes in eukaryotes. Bioessays 28, 913-922, doi:10.1002/bies.20452 (2006).

20 González, J., Lenkov, K., Lipatov, M., Macpherson, J. M. \& Petrov, D. A. High Rate of Recent Transposable Element-Induced Adaptation in Drosophila melanogaster. PLOS Biology 6, e251, doi:10.1371/journal.pbio.0060251 (2008).

21 Garcia-Perez, J. L. et al. LINE-1 retrotransposition in human embryonic stem cells. Hum Mol Genet 16, 1569-1577, doi:10.1093/hmg/ddm105 (2007).

22 Kano, H. et al. L1 retrotransposition occurs mainly in embryogenesis and creates somatic mosaicism. Genes Dev 23, 1303-1312, doi:10.1101/gad.1803909 (2009).

23 van den Hurk, J. A. et al. L1 retrotransposition can occur early in human embryonic development. Hum Mol Genet 16, 1587-1592, doi:10.1093/hmg/ddm108 (2007).

24 Wissing, S. et al. Reprogramming somatic cells into iPS cells activates LINE-1 retroelement mobility. Hum Mol Genet 21, 208-218, doi:10.1093/hmg/ddr455 (2012).

25 Korb, E. \& Finkbeiner, S. Arc in synaptic plasticity: from gene to behavior. Trends Neurosci 34, 591-598, doi:10.1016/j.tins.2011.08.007 (2011).

26 Plath, N. et al. Arc/Arg3.1 Is Essential for the Consolidation of Synaptic Plasticity and Memories. Neuron 52, 437-444, doi:https://doi.org/10.1016/j.neuron.2006.08.024 (2006).

27 Bramham, C. R. et al. The Arc of synaptic memory. Experimental brain research 200, 125140, doi:10.1007/s00221-009-1959-2 (2010).

28 Hunter, R. Stress, Transposons, and the Brain Epigenome. (2016).

29 Epel, E. S. et al. Accelerated telomere shortening in response to life stress. Proceedings of the National Academy of Sciences of the United States of America 101, 17312-17315, doi:10.1073/pnas.0407162101 (2004). 